${ }^{1}$ Peruan A., ${ }^{2}$ Rusu G.

\title{
ABSTRACT SECOND ORDER DIFFERENTIAL EQUATIONS WITH TWO SMALL PARAMETERS AND LIPSCHITZIAN NONLINEARITIES
}

\begin{abstract}
In a real Hilbert space $H$ we consider the following singularly perturbed Cauchy problem $\varepsilon u_{\varepsilon \delta}^{\prime \prime}(t)+\delta u_{\varepsilon \delta}^{\prime}(t)+A u_{\varepsilon \delta}(t)+B\left(u_{\varepsilon \delta}(t)\right)=f(t), \quad t \in(0, T), u_{\varepsilon \delta}(0)=u_{0}, u_{\varepsilon \delta}^{\prime}(0)=u_{1}$, where $u_{0}, u_{1} \in H, f:[0, T] \mapsto H, \varepsilon, \delta$ are two small parameters, $A$ is a linear self-adjoint operator and $B$ is a nonlinear lipschitzian operator. We study the behavior of solutions $u_{\varepsilon \delta}$ in two different cases: $\varepsilon \rightarrow 0$ and $\delta \geq \delta_{0}>0 ; \varepsilon \rightarrow 0$ and $\delta \rightarrow 0$, relative to solution to the corresponding unperturbed problem.

Key words and phrases: singular perturbation, abstract second order Cauchy problem, lipschitzian nonlinearity, a priori estimate.
\end{abstract}

\footnotetext{
${ }^{1}$ Moldova State University, Chishnau, Republic of Moldova,

${ }^{2}$ Moldova State University, Chishnau, Republic of Moldova

e-mail: ${ }^{1}$ aperjan1248@gmail.com, ${ }^{2}$ rusugalinamoldova@gmail.com
}

\section{INTRODUCTION}

Let $H$ be a real Hilbert space endowed with the scalar product $(\cdot, \cdot)$ and the norm $|\cdot|$. Let $A: D(A) \subset H \rightarrow H$, be a linear self-adjoint operator and $B$ is nonlinear lipschitzian opeartor. Consider the following Cauchy problem:

$$
\left\{\begin{array}{l}
\varepsilon u_{\varepsilon \delta}^{\prime \prime}(t)+. u_{\varepsilon \delta}^{\prime}(t)+A u_{\varepsilon \delta}(t)+B\left(u_{\varepsilon \delta}(t)\right)=f(t), \quad t \in(0, T) \\
u_{\varepsilon \delta}(0)=u_{0}, \quad u_{\varepsilon \delta}^{\prime}(0)=u_{1}
\end{array}\right.
$$

where $u_{0}, u_{1}, f:[0, T] \rightarrow H$ and $\varepsilon, \delta$ are two small parameters. We investigate the behavior of solutions $u_{\varepsilon \delta}$ to the problem $\left(P_{\varepsilon \delta}\right)$ in two different cases:

(i) $\varepsilon \rightarrow 0$ and $\delta \geq \delta_{0}>0$, relative to the solutions to the following unperturbed system:

$$
\left\{\begin{array}{l}
\delta l_{\delta}^{\prime}(t)+A l_{\delta}(t)+B\left(l_{\delta}(t)\right)=f(t), \quad t \in(0, T), \\
l_{\delta}(0)=u_{0}
\end{array}\right.
$$

УДК 517.9

2010 Mathematics Subject Classification: 34E15, 34G20, 35B25, 47 J35.

This research was supported by the State Program of the Republic of Moldova Multivalued dynamical systems, singular perturbations, integral operators and non-associative algebraic structures (20.80009.5007.25)

(C) ${ }^{1}$ Perjan A., ${ }^{2}$ Rusu G., 2020 
(ii) $\varepsilon \rightarrow 0$ and $\delta \rightarrow 0$, relative to the solutions to the following unperturbed system:

$$
A v(t)+B(v(t))=f(t), \quad t \in[0, T),
$$

The problem $\left(P_{\varepsilon \delta}\right)$ is the abstract model of singularly perturbed problems of hyperbolicparabolic type in the case (i) and of the hyperbolic-parabolic-elliptic type in the case (ii). Such kind of problems arise in various fields of science and technology, for example, in the mathematical modeling of elasto-plasticity phenomena.

In many works, under various restrictions singularly perturbed Cauchy problems for linear or nonlinear differential equations of second order of type $\left(P_{\varepsilon \delta}\right)$ were studied. Without pretending to a complete analysis, we will mention the works $[2,3,4,5,8,9]$, in which the reader can find an extensive bibliography.

In most of the mentioned cases the results were obtained by using the theory of semigroups of linear operators. Different to other methods, our approach is based on two key points. The first one is the relationship between solutions to the Cauchy problem for the abstract linear second order differential equation and the corresponding problem for the first order equation. The second key point are a priori estimates of solutions, which are uniform with respect to the small parameter. Moreover, we study the problem $\left(P_{\varepsilon \delta}\right)$ for a larger class of functions, i. e. $f \in W^{1, p}(0, T ; H)$. Also we obtain the convergence rate, as $\varepsilon \rightarrow 0$, which depends on $p$.

The organization of this paper is as follows. At the beginning of the next section we present the theorems of existence and uniqueness of solutions to the problems $\left(P_{\varepsilon \delta}\right),\left(P_{\delta}\right)$ and some a priori estimates of these solutions. Then we present a relationship between solutions to the problem for the abstract linear second order differential equation and the corresponding solution to the problem for the first order equation. In the section 3 we present the main result of the paper. More precisely, we prove the convergence estimates of the difference of solutions to the problems $\left(P_{\varepsilon \delta}\right)$ and $\left(P_{\delta}\right)$ for $\varepsilon \rightarrow 0, \delta \geq \delta_{0}>0$ and also to the problems $\left(P_{\varepsilon \delta}\right)$ and $\left(P_{0}\right)$ for $\varepsilon \rightarrow 0, \delta \rightarrow 0$.

In what follows we will need some notations. Let $k \in N^{*}, 1 \leq p \leq+\infty,(a, b) \subset$ $(-\infty,+\infty)$ and $X$ be a Banach space. By $W^{k, p}(a, b ; X)$ denote the Banach space of vectorial distributions $u \in D^{\prime}(a, b ; X), u^{(j)} \in L^{p}(a, b ; X), j=0,1, \ldots, k$, endowed with the norm

$$
\|u\|_{W^{k, p}(a, b ; X)}=\left\{\begin{array}{l}
\left(\sum_{j=0}^{k}\left\|u^{(j)}\right\|_{L^{p}(a, b ; X)}^{p}\right)^{\frac{1}{p}} \text { for } p \in[1, \infty), \\
\|u\|_{W^{k, \infty}(a, b ; X)}=\max _{0 \leq j \leq k}\left\|u^{(j)}\right\|_{L^{\infty}(a, b ; X)} \text { for } p=\infty .
\end{array}\right.
$$

If $X$ is a Hilbert space, then $W^{k, 2}(a, b ; X)$ is also a Hilbert space with the scalar product

$$
(u, v)_{H^{k}(a, b ; X)}=\sum_{j=0}^{k} \int_{a}^{b}\left(u^{(j)}(t), v^{(j)}(t)\right)_{X} d t .
$$

The framework of our paper will be determined by the following conditions:

(HA) The operator $A: D(A) \subset H \rightarrow H$ is linear, self-adjoint and positive definite, i.e. there exists $\omega>0$ such that $(A u, u) \geq \omega|u|^{2}, \quad \forall u \in D(A)$;

(HB) The operator $B: D(B) \subseteq H \mapsto H$ is lipschitzian, i. e. $D(A) \subset D(B)$ and there exists $L>0$ such that $|B(u)-B(v)| \leq L|u-v|, \quad \forall u, v \in D(B)$. 


\section{PRELIMINARIES}

In this section we remind results about the solvability of problems $\left(P_{\varepsilon \delta}\right),\left(P_{\delta}\right)$ and $\left(P_{0}\right)$. Since these results do not depend on the positive values of the parameters $\varepsilon$ and $\beta$, we will put $\varepsilon=\delta=1$. In this case

the problem $\left(P_{\varepsilon \delta}\right)$ takes the form:

$$
\left\{\begin{array}{l}
u^{\prime \prime}(t)+u^{\prime}(t)+A u(t)+B(u(t))=f(t), \quad t \in(0, T), \\
u(0)=u_{0} \in V, \quad u^{\prime}(0)=u_{1} \in H,
\end{array}\right.
$$

the problem $\left(P_{\delta}\right)$ takes the form:

$$
\left\{\begin{array}{l}
l^{\prime}(t)+A l(t)+B(l(t))=f(t), \quad t \in(0, T), \\
l(0)=u_{0} \in V
\end{array}\right.
$$

and the problem $\left(P_{0}\right)$ takes the form:

$$
A v+B(v)=f(t), \quad t \in(0, T) .
$$

The following theorems were inspired by the work [1] and are completely proved in the work [6].

Theorem 1. Let $T>0$. Assume that conditions (HA) and (HB) are fulfilled. If $u_{0} \in D(A)$, $u_{1} \in D\left(A^{1 / 2}\right)$ and $f \in W^{1,1}(0, T ; H)$, then there exists a unique function $u \in W^{2, \infty}(0, T ; H)$, $A^{1 / 2} u^{\prime} \in L^{2}(0, T ; H)$ and $A u \in L^{\infty}(0, T ; H)$ such that $u$ satisfies the equation (1) in the sence of distributions on $(0, T)$ and the initial conditions from (1). This function is called the strong solution to the problem (1).

Theorem 2. Let $T>0$. Assume that conditions (HA) and (HB) are fulfilled. If $u_{0} \in H$ and $f \in L^{2}(0, T ; H)$, then there exists a unique function $l \in C([0, T] ; H), A^{1 / 2} l \in L^{2}(0, T ; H)$ such that $l$ satisfies the equation (2) in the sence of distributions on $(0, T)$ and the initial condition from (2). This function is called the strong solution to the problem (2).

Theorem 3. Let $T>0$ and $p>1$. Suppose that conditions (HA) and (HB) are fulfilled and $\omega>L$. If $f \in W^{1, p}(0, T ; H)$, then the equation $A v+B(v)=f$ has a unique strong solution $v \in W^{1, p}(0, T ; H)$ and

$$
\|v\|_{W^{1, p}(0, T ; H)} \leq \frac{1}{q_{0}}\|f\|_{W^{1, p}(0, T ; H)}, \quad q_{0}=\omega-L .
$$

For the further consideration we rewrite the problems $\left(P_{\varepsilon \delta}\right)$ and $\left(P_{\delta}\right)$ in the form:

$$
\left\{\begin{array}{l}
\mu U_{\mu}^{\prime \prime}(s)+U_{\mu}^{\prime}(s)+A U_{\mu}(s)+B\left(U_{\mu}(s)\right)=F(s), \quad s \in(0, T / \delta), \\
U_{\mu}(0)=u_{0}, \quad U_{\mu}^{\prime}(0)=\delta u_{1},
\end{array}\right.
$$

and

$$
\left\{\begin{array}{l}
\mathcal{L}^{\prime}(s)+A \mathcal{L}(s)+B(\mathcal{L}(s))=F(s), \quad s \in(0, T / \delta) \\
\mathcal{L}(0)=u_{0}
\end{array}\right.
$$


where $U_{\mu}(s)=u_{\varepsilon \delta}(\delta s), \mathcal{L}(s)=l_{\delta}(s \delta), F(s)=f(s \delta)$ and $\mu=\varepsilon / \delta^{2}$.

In what follows we will prove some a priori estimates for solutions to the problems $\left(\mathcal{P}_{\mu}\right)$ and $\left(\mathcal{P}_{0}\right)$.

Lemma 1. Suppose that $q_{0}=\omega-L>0$ and conditions (HA) and (HB) are fulfilled. If $u_{0} \in D(A), u_{1} \in D\left(A^{1 / 2}\right), F \in W^{1,1}(0, \infty ; H)$ then for any strong solution $U_{\mu}$ to the problem $\left(\mathcal{P}_{\mu}\right)$ the following estimate

$$
\left\|U_{\mu}^{\prime}\right\|_{C([0, s]: H)} \leq C\left(q_{0}\right) \mathcal{M}_{0}(s), \quad s \geq 0, \quad \delta \in(0,1], \quad \mu \in\left(0, \mu_{0}\right],
$$

holds with $\mu_{0}=\frac{\omega-L}{2 L^{2}}$ and $\mathcal{M}_{0}(s)=\left|A u_{0}\right|+\left|A^{1 / 2} u_{1}\right|+|B(0)|+|F(0)|+|| F \|_{W^{1,1}(0, s ; H)}$.

Proof. Let $U_{\mu h}(s)=U_{\mu}(s+h)-U_{\mu}(s)$ and denote by

$$
\begin{aligned}
E\left(U_{\mu h}, s\right)= & \left|U_{\mu h}(s)\right|^{2}+\left|U_{\mu h}(s)+2 \mu U_{\mu h}^{\prime}(s)\right|^{2}+4 \mu\left(A U_{\mu h}(s), U_{\mu h}(s)\right)+ \\
& +4 \mu \int_{0}^{s}\left|U_{\mu h}^{\prime}(\tau)\right|^{2} d \tau+4 \int_{0}^{s}\left(A U_{\mu h}(\tau), U_{\mu}(\tau)\right) d \tau .
\end{aligned}
$$

If $U_{\mu}$ is a strong solution to the problem $\left(\mathcal{P}_{\mu}\right)$, then

$$
\frac{d}{d s} E\left(U_{\mu h}, s\right)=4\left(F_{h}(s)-\left(B\left(U_{\mu}(s)\right)\right)_{h}, U_{\mu h}(s)+2 \mu U_{\mu h}^{\prime}(s)\right), \quad s \geq 0 .
$$

Since

$$
\begin{gathered}
\left.\mid\left(B\left(U_{\mu}(s)\right)\right)_{h}, U_{\mu h}(s)+2 \mu U_{\mu h}^{\prime}(s)\right)\left.|\leq \mu| U_{\mu h}^{\prime}(s)\right|^{2}+L \omega^{-1}(1+L \mu)\left(A U_{\mu h}(s), U_{\mu h}(s)\right) \leq \\
\leq \mu\left|U_{\mu h}^{\prime}(s)\right|^{2}+\frac{L+\omega}{2 \omega}\left(A U_{\mu h}(s), U_{\mu h}(s)\right), \quad \mu \in\left(0, \mu_{0}\right] .
\end{gathered}
$$

then integrating $(6)$ on $(0, s)$, we get

$$
\begin{gathered}
\left|U_{\mu h}(s)\right|^{2}+\left|U_{\mu h}(s)+2 \mu U_{\mu h}^{\prime}(s)\right|^{2}+2 q_{0} \omega^{-1} \int_{0}^{s}\left(A U_{\mu h}(\tau), U_{\mu}(\tau)\right) d \tau \leq \\
\leq E\left(U_{\mu h}, 0\right)+4 \int_{0}^{s} \mid F_{h}\left(\tau|| U_{\mu h}(\tau)+2 \mu U_{\mu h}^{\prime}(\tau) \mid d \tau, \quad s \geq 0, \quad \text { for } \quad \mu \in\left(0, \mu_{0}\right] .\right.
\end{gathered}
$$

Applying Gronwall-Bellman's Lemma to the last inequality, we obtain

$$
\left|U_{\mu h}(s)\right| \leq C\left[E^{1 / 2}\left(U_{\mu h}, 0\right)+\int_{0}^{s}\left|F_{h}(\tau)\right| d \tau\right], \quad s \geq 0, \quad \mu \in\left(0, \mu_{0}\right] .
$$

Under the conditions of Lemma, due to the Theorem 1, we have that the following relations

$$
\left\{\begin{array}{l}
\left|h^{-1} U_{\mu h}(s)\right| \rightarrow\left|U_{\mu}^{\prime}(s)\right|, \quad h \rightarrow 0, \quad \text { in } \quad C([0, T]), \\
\left|h^{-1} U_{\mu h}(s)+2 \mu h^{-1} U_{\mu h}^{\prime}\right| \rightarrow\left|U_{\mu}^{\prime}(s)+2 \mu U_{\mu}^{\prime \prime}(s)\right|, \quad h \downarrow 0, \quad \text { a.e. } \quad s \in(0, T), \\
\left|h^{-1} A^{1 / 2}\left(U_{\mu h}(s)\right)\right|^{2} \rightarrow\left|A^{1 / 2}\left(U_{\mu}^{\prime}(s)\right)\right|^{2}, \quad h \downarrow 0, \quad \text { a.e. } \quad s \in(0, T), \\
h^{-2} E\left(U_{\mu h}, 0\right) \rightarrow\left|\delta u_{1}\right|^{2}+\left|2\left(F(0)-A u_{0}-B\left(u_{0}\right)\right)-\delta u_{1}\right|^{2}+4 \mu \delta^{2}\left|A^{1 / 2} u_{1}\right|^{2}, \quad h \downarrow 0,
\end{array}\right.
$$


hold. Taking into account the relations (8), we divide (7) by $h$ and then pass to the limit in the obtained inequality, to get the estimate (5).

To establish the relationship between solutions to the problems $\left(\mathcal{P}_{\mu}\right)$ and $\left(\mathcal{P}_{0}\right)$ in the linear case we will define the kernel of transformation realizing this relationship.

For $\mu>0$ denote by

$$
K(t, \tau, \mu)=\frac{1}{2 \sqrt{\pi} \mu}\left(K_{1}(t, \tau, \mu)+3 K_{2}(t, \tau, \mu)-2 K_{3}(t, \tau, \mu)\right), \quad \forall \mu>0,
$$

where

$$
\begin{gathered}
K_{1}(t, \tau, \mu)=\exp \left\{\frac{3 t-2 \tau}{4 \mu}\right\} \lambda\left(\frac{2 t-\tau}{2 \sqrt{\mu t}}\right), \quad K_{2}(t, \tau, \mu)=\exp \left\{\frac{3 t+6 \tau}{4 \mu}\right\} \lambda\left(\frac{2 t+\tau}{2 \sqrt{\mu t}}\right), \\
K_{3}(t, \tau, \mu)=\exp \left\{\frac{\tau}{\mu}\right\} \lambda\left(\frac{t+\tau}{2 \sqrt{\mu t}}\right), \quad \lambda(s)=\int_{s}^{\infty} e^{-\eta^{2}} d \eta .
\end{gathered}
$$

In the following lemma we present some properties of kernel $K(t, \tau, \mu)$, used in the proof of the following results.

Lemma 2. [7] The function $K(t, \tau, \mu)$ is solution to the problem

$$
\left\{\begin{array}{l}
K_{t}(t, \tau, \mu)=\mu K_{\tau \tau}(t, \tau, \mu)-K_{\tau}(t, \tau, \mu), \quad \forall t>0, \quad \forall \tau>0, \\
\mu K_{\tau}(t, 0, \mu)-K(t, 0, \mu)=0, \quad \forall t \geq 0 \\
K(0, \tau, \mu)=\frac{1}{2 \mu} \exp \left\{-\frac{\tau}{2 \mu}\right\}, \quad \forall \tau \geq 0,
\end{array}\right.
$$

from $C([0, \infty) \times[0, \infty)) \cap C^{2}((0, \infty) \times(0, \infty))$ and possesses the following properties:

(i) $K(t, \tau, \mu)>0, \quad \forall t \geq 0, \quad \forall \tau \geq 0, \quad$ and $\quad \int_{0}^{\infty} K(t, \tau, \mu) d \tau=1, \quad \forall t \geq 0$;

(ii) Let $q \in[0,1]$. Then $\int_{0}^{\infty} K(t, \tau, \mu)|t-\tau|^{q} d \tau \leq C(\mu+\sqrt{\mu t})^{q}, \quad \forall \mu>0, \quad \forall t \geq 0$;

(iii) Let $p \in(1, \infty]$ and $f:[0, \infty) \rightarrow H, f(t) \in W^{1, p}(0, \infty ; H)$. Then

$$
\left|f(t)-\int_{0}^{\infty} K(t, \tau, \mu) f(\tau) d \tau\right| \leq C(p)\left\|f^{\prime}\right\|_{L^{p}(0, \infty ; H)}(\mu+\sqrt{\mu t})^{\frac{p-1}{p}}, \quad \forall \mu>0, \quad \forall t \geq 0 ;
$$

Lemma 3. [7] Let $B=0$. Assume that $A: D(A) \subset H \rightarrow H$ is a linear, self-adjoint, positive definite operator and $F \in L^{\infty}(0, \infty ; H)$. If $U_{\mu}$ is a strong solution to the problem $\left(\mathcal{P}_{\mu}\right)$ with $U_{\mu} \in W^{2, \infty}(0, \infty ; H), A U_{\mu} \in L^{\infty}(0, \infty ; H)$, then the function $W_{\mu}$ defined by $W_{\mu}(s)=\int_{0}^{\infty} K(s, \tau, \mu) U_{\mu}(\tau) d \tau$ is the strong solution to the problem

$$
\left\{\begin{array}{l}
W_{\mu}^{\prime}(s)+A W_{\mu}(s)=F_{0}(s, \mu), \quad \text { a.e. } \quad s>0, \quad \text { in } \quad H \\
W_{\mu}(0)=\varphi_{\mu}
\end{array}\right.
$$

where

$$
\begin{gathered}
F_{0}(s, \mu)=\frac{1}{\sqrt{\pi}}\left[2 \exp \left\{\frac{3 s}{4 \mu}\right\} \lambda\left(\sqrt{\frac{s}{\mu}}\right)-\lambda\left(\frac{1}{2} \sqrt{\frac{s}{\mu}}\right)\right] u_{1}+\int_{0}^{\infty} K(s, \tau, \mu) F(\tau) d \tau \\
\varphi_{\mu}=\int_{0}^{\infty} e^{-\tau} U_{\mu}(2 \mu \tau) d \tau .
\end{gathered}
$$




\section{Behaviour of Solutions to The Problem $\left(P_{\varepsilon \delta}\right)$}

In this section we will prove the main result concerning the behavior of the solutions to the problem $\left(P_{\varepsilon \delta}\right)$, in both cases: $\varepsilon \rightarrow 0$ and $\delta \geq \delta_{0}>0 ; \varepsilon \rightarrow 0$ and $\delta \rightarrow 0$, relative to solution to the corresponding unperturbed problem.

Theorem 4. Let $T>0$ and $p \in(1, \infty]$. Assume that $q_{0}=\omega-L>0$ and conditions (HA) and $(\mathbf{H B})$ are fulfilled. If $u_{0} \in D(A), A^{1 / 2} u_{1} \in H$ and $f \in W^{1, p}(0, T ; H)$, then there exists constant $C=C(T, p, \omega, L)>0$ such that

$$
\left\|u_{\varepsilon \delta}-l_{\delta}\right\|_{C([0, T] ; H)} \leq C \mathcal{M} \varepsilon^{\beta} \delta^{-5 / 2}, \quad \delta \in(0,1], \quad \varepsilon \in\left(0, \mu_{0} \delta^{2}\right]
$$

where $u_{\varepsilon \delta}$ and $l_{\delta}$ are strong solutions to the problems $\left(P_{\varepsilon \delta}\right)$ and $\left(P_{\delta}\right)$, respectively, $\mu_{0}$ is defined in (5), $\mathcal{M}=\left|A u_{0}\right|+\left|A^{1 / 2} u_{1}\right|+|B(0)|+\|f\|_{W^{1,1}(0, T ; H)}$ and

$$
\beta=\left\{\begin{array}{l}
1 / 2 \text { if } f=0, \\
(p-1) /(2 p) \text { if } f \neq 0 .
\end{array}\right.
$$

Proof. During this proof we will agree to denote by $C$ all constants $C(T, p, \omega, L)$. For any $f \in W^{1, p}(0, T ; H)$ let us define the function $\widetilde{f}:[0, \infty) \mapsto H$ as follows:

$$
\widetilde{f}(t)=\left\{\begin{array}{l}
f(t), \quad 0 \leq t \leq T \\
\frac{2 T-t}{T} f(T), \quad T<t \leq 2 T \\
0, \quad t>2 T
\end{array}\right.
$$

Then $\tilde{f}(t) \in W^{1, p}(0, T ; H)$ and, since $W^{1, p}(0, T ; H) \hookrightarrow C([0, T] ; H)$ continuously, we get

$$
\|\widetilde{f}\|_{W^{1, p}(0, \infty ; H)} \leq C(p) \max \left\{T, \frac{1}{T}\right\}\|f\|_{W^{1, p}(0, T ; H)} .
$$

If we denote by $\widetilde{U}_{\mu}$ the unique strong solution to the problem $\left(\mathcal{P}_{\mu}\right)$, defined on $(0, \infty)$ instead of $(0, S)$ with $S=T / \delta$ and $\tilde{f}$ instead of $f$, then, from Theorem 1 and Lemma 1, it follows that $\tilde{U}_{\mu} \in W^{2, \infty}(0, \infty ; H), A^{1 / 2} \tilde{U}_{\mu}^{\prime} \in L^{2}(0, \infty ; H), A \tilde{U}_{\mu} \in L^{\infty}(0, \infty ; H)$.

Moreover, the estimate (11) implies

$$
\|\widetilde{F}\|_{W^{1, p}(0, \infty ; H)} \leq C(p, T) \delta^{-1 / p}\|f\|_{W^{1, p}(0, T ; H)}, \quad p \in(1, \infty), \forall \delta \in(0,1] .
$$

Due to the estimates (12) and Lemma 1, we obtain the following estimates

$$
\left\|\tilde{U}_{\mu}^{\prime}\right\|_{C([0, s] ; H)} \leq C \delta^{\gamma} \mathcal{M}, \quad s \geq 0, \quad \delta \in(0,1], \quad \mu \in\left(0, \mu_{0}\right] .
$$

holds with $\mathcal{M}$ from(9), $\mu_{0}$ from (5) and

$$
\gamma= \begin{cases}0 & \text { if } \quad f=0 \\ -1 / p & \text { if } \quad f \neq 0\end{cases}
$$


By Lemma 3 , the function $W_{\mu}$, defined by $W_{\mu}(s)=\int_{0}^{\infty} K(s, \tau, \mu) \widetilde{U}_{\mu}(\tau) d \tau$, is a strong solution to the problem

$$
\left\{\begin{array}{l}
W_{\mu}^{\prime}(s)+A W_{\mu}(s)=\widetilde{F}_{0}(s, \mu), \quad \text { a.e. } \quad s>0, \quad \text { in } \quad H \\
W_{\mu}(0)=\varphi_{\mu}
\end{array}\right.
$$

where

$$
\begin{gathered}
\widetilde{F}_{0}(s, \mu)=\delta f_{0}(s, \mu) u_{1}+\int_{0}^{\infty} K(s, \tau, \mu) \widetilde{F}(\tau) d \tau-\int_{0}^{\infty} K(s, \tau, \mu) B\left(\widetilde{U}_{\mu}(\tau)\right) d \tau \\
f_{0}(s, \mu)=\frac{1}{\sqrt{\pi}}\left[2 \exp \left\{\frac{3 s}{4 \mu}\right\} \lambda\left(\sqrt{\frac{s}{\mu}}\right)-\lambda\left(\frac{1}{2} \sqrt{\frac{s}{\mu}}\right)\right], \quad \varphi_{\mu}=\int_{0}^{\infty} e^{-\tau} \widetilde{U}_{\mu}(2 \mu \tau) d \tau .
\end{gathered}
$$

Denote by $R(s, \mu)=\widetilde{\mathcal{L}}(s)-W_{\mu}(s)$, where $\widetilde{\mathcal{L}}$ is the strong solution to the problem $\left(\mathcal{P}_{0}\right)$ with $\tilde{F}$ instead of $F, T=\infty$ and $W_{\mu}$ is the strong solution to the problem (15). Then, due to Theorem $2, R(\cdot, \mu) \in W_{\text {loc }}^{1, \infty}(0, \infty ; H)$ and $R$ is a strong solution in $H$ to the problem

$$
\left\{\begin{array}{l}
R^{\prime}(s, \mu)+A R(s, \mu)+B(\widetilde{\mathcal{L}}(s))-B\left(W_{\mu}(s)\right)=\mathcal{F}(s, \mu), \quad \text { a. e. } \quad s>0 \\
R(0, \mu)=u_{0}-W_{\mu}(0)
\end{array}\right.
$$

where

$$
\begin{gathered}
\mathcal{F}(s, \mu)=\tilde{F}(s)-\int_{0}^{\infty} K(s, \tau, \mu) \tilde{F}(\tau) d \tau-\delta f_{0}(s, \mu) u_{1}+ \\
+B\left(\widetilde{U}_{\mu}(s)\right)-B\left(W_{\mu}(s)\right)+\int_{0}^{\infty} K(s, \tau, \mu)\left[B\left(\widetilde{U}_{\mu}(\tau)\right)-B\left(\widetilde{U}_{\mu}(s)\right)\right] d \tau .
\end{gathered}
$$

In what follows we need the following two Lemmas, which will be proved after the proof of the Theorem 4 .

Lemma 4. Assume the conditions of Theorem 4 are fulfilled. Then the following estimates:

$$
\left|\widetilde{U}_{\mu}(s)-W_{\mu}(s)\right| \leq C \mathcal{M} \mu^{1 / 2} \delta^{\gamma}(1+\sqrt{s}), \quad s \geq 0, \delta \in(0,1], \mu \in\left(0, \mu_{0}\right]
$$

are valid with $\mathcal{M}$ from (9), $\gamma$ from (14) and $\mu_{0}$ from (5).

Lemma 5. Assume the conditions of Theorem 4 are fulfilled. Then for the strong solution to the problem (17) the following estimate

$$
\|R\|_{C([0, s] ; H)} \leq C \mathcal{M} \mu^{\beta} \delta^{\gamma}(1+\sqrt{s}) s, \quad s \geq 0, \quad \delta \in(0,1], \quad \mu \in\left(0, \mu_{0}\right],
$$

is true with $\mathcal{M}$ from(9), $\beta$ and $\gamma$ from(14) and $\mu_{0}$ from (5).

Finally, from these lemmas we deduce that

$$
\begin{aligned}
& \quad\left\|\tilde{U}_{\mu}-\tilde{L}\right\|_{C([0, s] ; H)} \leq\left\|\tilde{U}_{\mu}-W_{\mu}\right\|_{C([0, s] ; H)}+\|R\|_{C([0, s] ; H)} \leq \\
& \leq C \mathcal{M} \mu^{\beta} \delta^{\gamma}\left(1+s^{3 / 2}\right), \quad s \geq 0, \quad \delta \in(0,1], \quad \mu \in\left(0, \mu_{0}\right],
\end{aligned}
$$


Since $U_{\mu}(s)=\tilde{U}_{\mu}(s), L(s)=\tilde{L}(s)$, for all $s \in[0, T / \delta], U_{\mu}(s)=u_{\varepsilon \delta}(\delta s)$ and $L(s)=l_{\delta}(\delta s)$, then we have

$$
\begin{aligned}
& \left|u_{\varepsilon \delta}(t)-l_{\delta}(t)\right|=\left|u_{\varepsilon \delta}(\delta s)-l_{\delta}(\delta s)\right|=\left|\tilde{U}_{\mu}(s)-\tilde{L}(s)\right| \leq \\
& \leq C \mathcal{M} \mu^{\beta} \delta^{\gamma-3 / 2}, \quad t \in[0, T], \quad \delta \in(0,1], \quad \mu \in\left(0, \mu_{0}\right] .
\end{aligned}
$$

Concequently, from (21) follows the estimate (9). Theorem 4 is proved.

Proof of Lemma 4. Using properties (i) and (ii) from Lemma 2 and the estimate (13), we get

$$
\begin{gathered}
\left|\widetilde{U}_{\mu}(s)-W_{\mu}(s)\right| \leq \int_{0}^{\infty} K(s, \tau, \mu)\left|\widetilde{U}_{\mu}(s)-\widetilde{U}_{\mu}(\tau)\right| d \tau \leq \\
\leq \int_{0}^{\infty} K(s, \tau, \mu)\left|\int_{\tau}^{s}\right| \widetilde{U}_{\mu}^{\prime}(\xi)|d \xi| d \tau \leq C \mathcal{M} \delta^{\gamma} \int_{0}^{\infty} K(s, \tau, \mu)|\tau-s| d \tau \leq \\
\leq C \mathcal{M} \mu^{1 / 2} \delta^{\gamma}(1+\sqrt{s}), \quad s \geq 0, \quad \delta \in(0,1], \quad \mu \in\left(0, \mu_{0}\right] .
\end{gathered}
$$

Thus, Lemma 4 is proved.

Proof of Lemma 5. Multiplying scalarly in $H$ the equation (17) by $R$ and then integrating on $(0, s)$ the obtained equality, we deduce

$$
|R(s, \mu)|^{2}+2 q_{0} \int_{0}^{s}|R(\xi, \mu)|^{2} d \xi \leq|R(0, \mu)|^{2}+2 \int_{0}^{s}|\mathcal{F}(\xi, \mu)||R(\xi, \mu)| d \xi, \quad \forall s \geq 0,
$$

where $\mathcal{F}(\xi, \mu)$ is defined by (18). Applying Gronwall-Bellman's Lemma to the last inequality, we have that

$$
|R(s, \mu)| \leq|R(0, \mu)|+\int_{0}^{s}|\mathcal{F}(\xi, \mu)| d \xi, \quad \forall s \geq 0 .
$$

In what follows, we will estimate the right side of (22). Using (13), we get

$$
\begin{aligned}
& |R(0, \mu)| \leq \int_{0}^{\infty} e^{-\tau}\left|\tilde{U}_{\mu}(2 \mu \tau)-u_{0}\right| d \tau \leq \int_{0}^{\infty} e^{-\tau} \int_{0}^{2 \mu \tau}\left|\tilde{U}_{\mu}^{\prime}(\xi)\right| d \xi d \tau \leq \\
& \leq C \mathcal{M} \mu \delta^{\gamma} \int_{0}^{\infty} \tau e^{-\tau} d \tau=C \mathcal{M} \mu \delta^{\gamma}, \quad \delta \in(0,1], \quad \mu \in\left(0, \mu_{0}\right] .
\end{aligned}
$$

Let us estimate $|\mathcal{F}(t, \mu)|$. Using the property (iii) from Lemma 2 and (12), we have

$$
\begin{aligned}
& \left|\tilde{F}(s)-\int_{0}^{\infty} K(s, \tau, \mu) \tilde{F}(\tau) d \tau\right| \leq C\left\|\tilde{F}^{\prime}\right\|_{L^{p}(0, \infty ; H)}(\mu+\sqrt{\mu s})^{(p-1) / p} \leq \\
& \leq C\left\|\tilde{f}^{\prime}\right\|_{L^{p}(0, T ; H)}(\delta(\mu+\sqrt{\mu s}))^{(p-1) / p}, \quad s \geq 0, \quad \delta \in(0,1], \quad \mu>0 .
\end{aligned}
$$

Since $e^{\xi} \lambda(\sqrt{\xi}) \leq C, \quad \forall \xi \geq 0$, then the following estimates

$$
\begin{gathered}
\int_{0}^{s} \exp \left\{\frac{3 \xi}{4 \mu}\right\} \lambda\left(\sqrt{\frac{\xi}{\mu}}\right) d \xi \leq C \mu \int_{0}^{\infty} e^{-\xi / 4} d \xi \leq C \mu, \quad s \geq 0, \quad \mu>0, \\
\int_{0}^{s} \lambda\left(\frac{1}{2} \sqrt{\frac{\xi}{\mu}}\right) d \xi \leq \mu \int_{0}^{\infty} \lambda\left(\frac{1}{2} \sqrt{\xi}\right) d \xi \leq C \mu, \quad s \geq 0, \quad \mu>0
\end{gathered}
$$


hold. Consequently

$$
\left|\delta \int_{0}^{s} f_{0}(\xi, \mu) u_{1} d \xi\right| \leq C \delta \mu\left|u_{1}\right|, \quad s \geq 0, \quad \mu>0, \quad \delta>0 .
$$

Using the estimates (19), we get the following estimates

$$
\begin{gathered}
\quad\left|B\left(\widetilde{U}_{\mu}(s)\right)-B\left(W_{\mu}(s)\right)\right| \leq L\left|\widetilde{U}_{\mu}(s)-W_{\mu}(s)\right| \leq \\
\leq C \mathcal{M} \mu^{1 / 2} \delta^{\gamma}(1+\sqrt{s}) \quad s \geq 0, \quad \delta \in(0,1], \quad \mu \in\left(0, \mu_{0}\right], \\
\quad \int_{0}^{\infty} K(s, \tau, \mu)\left|B\left(\widetilde{U}_{\mu}(\tau)\right)-B\left(\widetilde{U}_{\mu}(s)\right)\right| d \tau \leq \\
\leq C \mathcal{M} \mu^{1 / 2} \delta^{\gamma}(1+\sqrt{s}) \quad s \geq 0, \quad \delta \in(0,1], \quad \mu \in\left(0, \mu_{0}\right] .
\end{gathered}
$$

Using (24), (25), (26) and (27), from (18) we obtain

$$
|\mathcal{F}(\tau, \mu)| \leq C \mathcal{M} \delta^{\gamma} \mu^{\beta}(1+\sqrt{s}), \quad s \geq 0, \delta \in(0,1], \quad \mu \in\left(0, \mu_{0}\right] .
$$

Consequently,

$$
\int_{0}^{s}|\mathcal{F}(\tau, \mu)| d \tau \leq C \mathcal{M} \delta^{\gamma} \mu^{\beta} s(1+\sqrt{s}), \quad s \geq 0, \delta \in(0,1], \quad \mu \in\left(0, \mu_{0}\right] .
$$

From (22), using (23) and (28) we get the estimate (20). Lemma 5 is proved.

Corollary 1. If the conditions of Theorem 4 are fulfilled and $\delta \geq \delta_{0}>0$, then

$$
\left\|u_{\varepsilon \delta}-l_{\delta}\right\|_{C([0, T] ; H)} \leq C \mathcal{M} \varepsilon^{\beta}
$$

with $C=C\left(T, p, \omega, L, \delta_{0}\right)>0, \mathcal{M}$ from (9) and $\beta$ from (10).

In what follows we will investigate the behavior of solutions to the problem $\left(P_{\delta}\right)$ as $\delta \rightarrow 0$.

Theorem 5. Let $T>0$ and $p \in(1, \infty]$. Assume that $q_{0}=\omega-L>0$ and conditions (HA) and (HB) are fulfilled. If $u_{0} \in D(A)$ and $f \in W^{1,1}(0, T ; H)$, then there exists constant $C=C(T, p, \omega, L)>0$ such that

$$
\left|l_{\delta}(t)-v(t)\right| \leq h_{0} e^{-q_{0} t / \delta}+C \delta^{(p-1) / p}|| f \|_{W^{1, p}(0, T ; H)}, \quad t \in(0, T], \quad \delta \in(0,1),
$$

where $l_{\delta}$ and $v$ are strong solutions to the problems $\left(P_{\delta}\right)$ and $\left(P_{0}\right)$, respectively, and $h_{0}=\left|u_{0}-(A+B)^{-1} f(0)\right|$.

Proof. Denote by $R_{1}(t, \delta)=l_{\delta}(t)-v(t)$. Then $R_{1}(t, \delta)$ is a strong solution to the problem

$$
\left\{\begin{array}{l}
\delta R_{1}^{\prime}(t, \delta)+A R_{1}(t, \delta)=-\delta v^{\prime}(t)-B\left(l_{\delta}(t)\right)+B(v(t)), \quad t \in(0, T), \\
R_{1}(0, \delta)=u_{0}-(A+B)^{-1} f(0),
\end{array}\right.
$$


Multiplying equation from (30) scalarly in $H$ by $R_{1}$, we obtain the equality

$$
\begin{gathered}
\delta \frac{d}{d t}\left|R_{1}(t, \delta)\right|^{2}+2\left(A R_{1}(t, \delta), R_{1}(t, \delta)\right)= \\
=-2 \delta\left(v^{\prime}(t), R_{1}(t, \delta)\right)+2\left(B(v(t))-B\left(l_{\delta}(t)\right), R_{1}(t, \delta)\right), \quad t \in(0, T) .
\end{gathered}
$$

Then, using conditions (HA), (HB) and $\omega-L>0$ and, we get

$$
\delta \frac{d}{d t}\left|R_{1}(t, \delta)\right|^{2}+2 q_{0}\left|R_{1}(t, \delta)\right|^{2} \leq 2 \delta\left|v^{\prime}(t)\right|\left|R_{1}(t, \delta)\right|, \quad t \in(0, T),
$$

or

$$
\frac{d}{d t}\left|R_{1}(t, \delta) e^{q_{0} t / \delta}\right|^{2} \leq 2\left|v^{\prime}(t) e^{q_{0} t / \delta}\right|\left|R_{1}(t, \delta) e^{q_{0} t / \delta}\right|, \quad t \in(0, T) .
$$

Integrating this inequality on $(0, t)$, we obtain

$$
\left|R_{1}(t, \delta) e^{q_{0} t / \delta}\right|^{2} \leq\left|R_{1}(0, \delta)\right|^{2}+2 \int_{0}^{t}\left|v^{\prime}(\tau) e^{q_{0} \tau / \delta}\right|\left|R_{1}(\tau, \delta) e^{q_{0} \tau / \delta}\right| d \tau, \quad t \in[0, T] .
$$

Applying Gronwall-Bellman's Lemma to the last inequality and using (4), we get the estimate

$$
\begin{gathered}
\left|R_{1}(t, \delta)\right| \leq\left|R_{1}(0, \delta)\right| e^{-q_{0} t / \delta}+\int_{0}^{t} e^{-q_{0}(t-\tau) / \delta}\left|v^{\prime}(\tau)\right| d \tau \leq \\
\leq\left|R_{1}(0, \delta)\right| e^{-q_{0} t / \delta}+\left(\frac{\delta}{q_{0}}\right)^{(p-1) / p}\left\|v^{\prime}\right\|_{L^{p}(0, T ; H)} \leq \\
\leq\left|R_{1}(0, \delta)\right| e^{-q_{0} t / \delta}+C\left(\frac{\delta}{q_{0}}\right)^{(p-1) / p}\|f\|_{W^{1, p}(0, T ; H)}, \quad t \in[0, T] .
\end{gathered}
$$

from which follows (29). Theorem 5 is proved.

Remark 1. Under the conditions of Theorem 5 it follows that for every $t_{0}>0$ and $T>t_{0}$,

$$
l_{\delta}(t) \rightarrow v(t) \quad \text { in } \quad C\left(\left[t_{0}, T\right] ; H\right) \quad \text { as } \quad \delta \rightarrow 0 .
$$

If the concordance condition $f(0)=A u_{0}+B\left(u_{0}\right)$ is satisfied, then $l_{\delta}(t) \rightarrow v(t)$ in $C([0, T] ; H)$, as $\delta \rightarrow 0$.

From Theorems 4 and 5 immediately follows the following theorem.

Theorem 6. Let $T>0$ and $p \in(1, \infty]$. Assume that $q_{0}=\omega-L>0$ and conditions (HA), (HB) are fulfilled. If $u_{0} \in D(A), u_{1} \in D\left(A^{1 / 2}\right)$ and $f \in W^{1, p}(0, T ; H)$, then there exists constant $C=C(T, p, \omega, L)>0$ such that

$$
\left\|u_{\varepsilon \delta}-v\right\|_{C([0, T] ; H)} \leq h_{0} e^{-q_{0} t / \delta}+C \mathcal{M} \Theta(\varepsilon, \delta), \quad \delta \in(0,1], \varepsilon \in\left(0, \mu_{0} \delta^{2}\right],
$$

where $u_{\varepsilon \delta}$ and $v$ are strong solutions to the problems $\left(P_{\varepsilon \delta}\right)$ and $\left(P_{0}\right)$, respectively, $\mathcal{M}$ is from (9), $h_{0}=\left|u_{0}-(A+B)^{-1} f(0)\right|, \mu_{0}$ is from (5) and

$$
\Theta=\left\{\begin{array}{l}
\varepsilon^{1 / 2} \delta^{-5 / 2}, \quad \text { if } \quad f=0, \\
\delta^{(p-1) / p}+\varepsilon^{(p-1 /(2 p)} \delta^{-5 / 2}, \quad \text { if } f \neq 0 .
\end{array}\right.
$$

Remark 2. Under the conditions of Theorem 6 it follows that for every $t_{0}>0$ and $T>t_{0}$,

$$
u_{\varepsilon \delta}(t) \rightarrow v(t) \quad \text { in } \quad C\left(\left[t_{0}, T\right] ; H\right) \quad \text { as } \quad \Theta(\varepsilon, \mu) \rightarrow 0 .
$$

If the concordance condition $f(0)=A u_{0}+B\left(u_{0}\right)$ is satisfied, then $u_{\varepsilon \delta}(t) \rightarrow v(t)$ in $C([0, T] ; H)$, as $\Theta \rightarrow 0$. 


\section{REFERENCES}

[1] V. Barbu, Nonlinear Differential Equations of Monotone Types in Banach Spaces, Springer-Verlag, New York, 2010.

[2] K. J. Engel, On singular perturbations of second order Cauchy problems, Pacific J. Math., 152(1992), no. 1, 79-91.

[3] H. O. Fattorini, The hyperbolic singular perturbation problem: an operator approach, J. Differential Equations, 70(1987), no. 1, 1-41.

[4] M. Ghisi and M. Gobbino Global-in-time uniform convergence for linear M.hyperbolic-parabolic singular perturbations, Acta Math. Sinica (English Series), 22(2006), no. 4, 1161-1170.

[5] B. Najman, Time singular limit of semilinear wave equations with damping, J. Math. Anal. Appl., 174, (1993), 95-117.

[6] A. Perjan, Singularly perturbed boundary value problems for evolution differential equations, D.Sc. thesis, Moldova State University, 2008.

[7] A. Perjan, Linear singular perturbations of hyperbolic-parabolic type, Bul. Acad. Stiinte Repub. Mold. Mat., 42(2003), no. 2, 95-112.

[8] A. Perjan and G. Rusu, Convergence estimates for abstract second-order singularly perturbed Cauchy problems with Lipschitz nonlinearities, Asymptot. Anal. 97(2016), no. 3-4, 337-349.

[9] A. Perjan and G. Rusu, Convergence estimates for abstract second order differential equations with two small parameters and monotone nonlinearities, Topol. Methods Nonlinear Anal., 54(2019), no. 2B, 1093-1110.

Received 29.10.2020

Пержан А., Русу Г. Абстрактні диференціальні рівняння другого порядку з двома малими параметрами $і$ ліпшіцевими нелінійностлми // Буковинський матем. журнал - 2020. T.8, №1. - C. 29-40.

У дійсному гільбертовому просторі $H$ розглянуто таку сингулярно збурену задачу Коші

$$
\left\{\begin{array}{l}
\varepsilon u_{\varepsilon \delta}^{\prime \prime}(t)+. u_{\varepsilon \delta}^{\prime}(t)+A u_{\varepsilon \delta}(t)+B\left(u_{\varepsilon \delta}(t)\right)=f(t), \quad t \in(0, T) \\
u_{\varepsilon \delta}(0)=u_{0}, \quad u_{\varepsilon \delta}^{\prime}(0)=u_{1}
\end{array}\right.
$$

де $u_{0}, u_{1} \in H, f:[0, T] \rightarrow H$ и $\varepsilon, \delta$ - малі параметри. Досліджено поведінку розв'язків $u_{\varepsilon \delta}$ задачи $\left(P_{\varepsilon \delta}\right)$ в у двох випадках:

$(i) \varepsilon \rightarrow 0$ и $\delta \geq \delta_{0}>0$, відносно розв'язків такої незбуреної задачі:

$$
\left\{\begin{array}{l}
\delta l_{\delta}^{\prime}(t)+A l_{\delta}(t)+B\left(l_{\delta}(t)\right)=f(t), \quad t \in(0, T), \\
l_{\delta}(0)=u_{0}
\end{array}\right.
$$

(ii) $\varepsilon \rightarrow 0$ и $\delta \rightarrow 0$, відносно розв'язків незбуреної задачі:

$$
A v(t)+B(v(t))=f(t), \quad t \in[0, T)
$$

Задача $\left(P_{\varepsilon \delta}\right)$ є абстрактною моделлю сингулярно збурених задач гіперболічно-параболічног типу у випадку (i) і гіперболічно-параболічно-еліптичного типу у випадку (ii). Подібні задачі виникають у різноманітних областях науки і техніки. 
На відміну від інших методів, даний метод грунтується на двох ключових позиціях. По-перше, одержано формулу, яка зв'язує розв'язок задачі Коші для абстрактного лінійного диференціального рівняння другого порядку з відповідним розв'язком задачі для рівняння першого порядку. По-друге, отримано it апріорні оцінки розв'язків, які є рівномірними щодо малого параметра. Крім того, досліджено задачу $\left(P_{\varepsilon \delta}\right)$ для ширшого класу функцій, а саме $f \in W^{1, p}(0, T ; H)$. Також встановлено швидкість збіжності розв'язком, яка залежить від $p$, при $\varepsilon \rightarrow 0$ и $\delta \rightarrow 0$. 